\section{Comparação do registro da produção ambulatorial em oncologia no Sistema Único de Saúde}

\author{
A comparative analysis of the ambulatory care \\ production register in oncology in the Brazilian \\ Unified Health System
}

Saint Clair S. Gomes Jr. 1

Rosimary T. Almeida 1
1 Instituto Alberto Luiz Coimbra de Pós-graduação e Pesquisa de Engenharia, Universidade Federal do Rio de Janeiro,

Rio de Janeiro, Brasil.

Correspondência S. C. S. Gomes Jr.

Programa de Engenharia Biomédica, Instituto Alberto Luiz Coimbra de

Pós-graduação e Pesquisa de Engenharia, Universidade Federal do Rio de Janeiro. C.P. 68.510,

Cidade Universitária,

Rio de Janeiro, $R J$ 21945-970, Brasil. saint@peb.ufrj.br

\section{Abstract}

The response of healthcare services to new information systems depends upon, among other factors, their local organization. This study aimed at comparing the chemotherapy and $\mathrm{ra}$ diotherapy production registers for the cities Rio de Janeiro and Belo Horizonte, Brazil, in the Ambulatory Information System of the Unified Brazilian Health System (in Portuguese, SUS) between November/1998 and February/2003. Both cities showed a pattern of increase for the chemotherapy production series, while the radiotherapy series showed such increase only for Rio de Janeiro. Comparing Box-Jenkins models for each series, Rio de Janeiro showed a delay of three months in recording production, due to public services. In Belo Horizonte, the delay was shorter than in Rio de Janeiro and there was no difference between public and non-public services. The observed differences between the cities seem to be associated with the fact that Belo Horizonte already had a mechanism to control and evaluate production before the introduction of the system in 1998.

Time Series; Ambulatory Care Information Systems; Medical Oncology

\section{Introdução}

O controle do câncer envolve um conjunto de ações nas mais diversas áreas. Uma dessas áreas é a assistência oncológica, que, devido ao uso de tecnologias de alta complexidade e custo, demanda consideráveis recursos do Sistema Único de Saúde (SUS) 1. Percebendo a necessidade de criar mecanismos de controle e avaliação da assistência oncológica prestada à população, o Ministério da Saúde (MS), em parceria com o Instituto Nacional de Câncer (INCA), elaborou o Programa de Avaliação e Controle em Oncologia, pioneiramente implementado nos municípios de Belo Horizonte, Minas Gerais, e Campos dos Goytacazes, Rio de Janeiro. Como principais pontos desse programa, podese destacar: auditorias permanentes nos serviços de assistência oncológica, treinamento dos funcionários desses serviços para o preenchimento dos novos formulários de controle da produção realizada, incentivo ao desenvolvimento de sistemas de informação específicos para o faturamento dos procedimentos oncológicos e outros 2 .

A experiência adquirida durante a implantação do Programa de Avaliação e Controle em Oncologia serviu de base para uma completa reformulação do sistema de cobrança dos procedimentos oncológicos junto ao SUS, oficializada pelas portarias GM/MS 3.535 (Diário Oficial da União 1998; 14 out) e GM/MS 3.536 (Diá- 
rio Oficial da União 1998; 14 out). As principais ações para essa reformulação foram: inclusão de normas e procedimentos para o cadastramento de um prestador de serviço como Centro de Alta Complexidade em Oncologia (CACON); alteração nas descrições e valores dos procedimentos de quimioterapia e de radioterapia; incorporação definitiva dos procedimentos de quimioterapia e de radioterapia ao Sistema de Informação das Autorizações de Procedimentos de Alta Complexidade (APACSIA/SUS) e outras 3 .

A reformulação também implementou a necessidade de autorização prévia para a realização dos procedimentos de quimioterapia e de radioterapia. Essa autorização é concedida mediante a apresentação do laudo de solicitação de procedimentos oncológicos. As informações desse laudo são parcialmente repassadas ao formulário de Autorização de Procedimentos de Alta Complexidade em Oncologia (APAC-Oncologia) e, posteriormente, sistematizadas na base de dados do módulo de oncologia do APACSIA/SUS. Esse módulo é utilizado para controlar o reembolso da produção de quimioterapia e de radioterapia realizada pelas unidades prestadoras de serviço do SUS. O módulo de oncologia do APACSIA/SUS armazena, além de dados de produção, uma série de dados de interesse clínico-epidemiológico, tais como: localização primária do tumor, estadiamento, finalidade do tratamento etc. 3 .

A associação dos dados armazenados no APACSIA/SUS a outros conjuntos de dados disponíveis no SUS (produção hospitalar, mortalidade etc.) torna-se um importante ingrediente na construção de indicadores de avaliação e controle das políticas públicas de controle e prevenção do câncer 4. Contudo, antes de obterse tais indicadores, torna-se necessário avaliar a qualidade do dado armazenado. Essa avaliação envolve uma seqüência de análises que abordam desde os atributos técnicos do sistema, passando pela capacidade do usuário em responder adequadamente ao sistema, até os impactos resultantes de sua utilização 5 .

Tendo essa motivação, este trabalho visa comparar o padrão de registro dos dados da produção ambulatorial de quimioterapia e de radioterapia dos serviços de dois municípios com demandas semelhantes, porém com diferentes experiências de implantação do módulo de oncologia do APACSIA/SUS. Com isso, busca-se avaliar o potencial dos dados de cada uma dessas realidades na construção de instrumentos de apoio ao planejamento e gestão de políticas de prevenção e controle do câncer.

\section{Materiais e métodos}

Para este trabalho, foram utilizados dados da produção de serviços oncológicos dos municípios do Rio de Janeiro e de Belo Horizonte, faturados no período de novembro de 1998 (mês de implantação do módulo de oncologia no APACSIA/SUS) a fevereiro de 2003. Esses municípios foram selecionados por apresentarem similaridade no perfil epidemiológico da população, serem centros de referência para seus respectivos Estados e, ainda, por apresentarem processos diferenciados de implantação do módulo de oncologia do APACSIA/SUS. Quanto a esse último aspecto, cabe ressaltar que o $\mathrm{Mu}$ nicípio de Belo Horizonte, diferentemente do Rio de Janeiro, participou do Programa de Avaliação e Controle em Oncologia e possuía, desde 1993, uma política de avaliação e controle da produção de procedimentos de alta complexidade 6 . Por outro lado, o Município do Rio de Janeiro destaca-se por apresentar a maior rede pública de prestadores de serviço do país, incluindo as três unidades de serviço do INCA 7.

As séries históricas utilizadas neste trabalho foram construídas a partir de dados disponibilizados pelo DATASUS (Departamento de Informática do SUS), no seguinte endereço eletrônico: ftp://msbbs.datasus.gov.br/Arquivos_ Publicos/. Todo o processo de manipulação desses dados e a construção de um arquivo de dados integrando diferentes períodos de faturamento são descritos em trabalho anterior 8 . Após a obtenção da base de dados para a realização deste trabalho, foram selecionadas as seguintes variáveis:

- Data de competência da APAC: data que define o período de faturamento da APAC-Oncologia;

- Tipo de tratamento realizado: quimioterapia ou radioterapia;

- Tipo de prestador de serviço: público (rede própria, municipal, estadual e federal ou unidades universitárias financiadas pelo setor público), contratado (unidades privadas, inclusive universitárias, e filantrópicas conveniadas ao SUS);

- Município no qual o procedimento foi realizado: Belo Horizonte ou Rio de Janeiro.

As séries históricas, estudadas neste trabalho, representam a quantidade de APAC-Oncologia faturadas mensalmente pelos prestadores de serviço de cada município. A apresentação mensal da produção realizada de quimioterapia e de radioterapia é o que garante o ressarcimento do tratamento prestado aos casos de câncer para os prestadores de serviços oncológicos do SUS (públicos e contratados). Devido 
a essa característica, os prestadores de serviço apresentam diferentes volumes mensais de produção, o que prejudicaria as comparações. Para poder comparar prestadores de serviço com diferentes volumes de produção, optou-se por padronizar cada uma das séries pelos seus respectivos valores máximos observados. A padronização realizada apenas reduz a escala dos valores da variável observada (quantidade de APAC-Oncologia apresentada no mês) para o intervalo entre zero e um, não alterando, entretanto, o padrão de distribuição dos valores da série.

Para descrever e modelar eventuais padrões de tendência, sazonalidade e, também, a estrutura de dependência temporal da série, foi escolhida a técnica de Box-Jenkins. De acordo com Moretin \& Toloi 9 , essa técnica envolve um ciclo interativo baseado em três estágios, que são: - Identificação: realizada com auxílio de gráficos dos dados brutos e das funções de autocorrelação (FAC) e autocorrelação parcial (FACP). A análise da FAC e da FACP auxilia na identificação da estrutura de formação da série, que pode ser: auto-regressiva (AR) - quando o modelo descreve uma série cujo valor presente é uma combinação linear de "p" valores defasados da própria série; média-móvel (MA) - quando o modelo descreve uma série cujo valor presente é uma combinação linear de "q" diferenças passadas dos valores observados em relação ao valor esperado para o mesmo período observado (componente aleatório, ruído branco ou choque aleatório); ou uma combinação das estruturas AR e MA (ARMA). A análise da FAC e da FACP também auxilia na definição do número máximo de parâmetros "p" e "q" que devem ser incorporados ao modelo 10;

- Estimação: obtenção dos coeficientes de ajuste do modelo de Box-Jenkins. Para estimar esses coeficientes, foi utilizada a função de verossimilhança dos dados observados. Métodos tradicionais de ajuste de modelos lineares (MQO: Mínimos Quadrados Ordinários; e MQG: Mínimos Quadrados Generalizados) não são adequados, devido à presença de autocorrelação serial do erro aleatório 11,12.

- Diagnóstico: seleção do "melhor modelo" por meio do critério de parcimônia, ou seja, melhor ajuste com o mínimo de parâmetros. $\mathrm{O}$ diagnóstico de ajuste dos modelos de Box-Jenkins foi realizado por meio da análise dos coeficientes AIC (Akaike's Information Criterion) e SBC (Schwars's Bayesian Criterion). Esses coeficientes utilizam, para a escolha do "melhor modelo", informação da função de verossimilhança, do número de parâmetros e do tamanho da amostra utilizada. Quanto menor os va- lores de AIC e SBC, melhor o ajuste do modelo proposto aos dados observados 10 .

Dois pontos devem ser observados durante o processo de ajuste de uma série temporal ao modelo de Box-Jenkins: período de observação, que deve ser grande ( $n>50)$, e distribuição dos valores observados, que deve estar em torno de um valor médio constante. A última condição garante a estabilidade da série. Para séries não-estacionárias, é necessário que sejam aplicadas técnicas de estabilização, tais como: diferenças sucessivas da série original, aplicação da função logaritmo, transformação de Box-Cox etc. 9 . A técnica de estabilização escolhida foi a de diferenças sucessivas, que, além da fácil implementação, apresenta bons resultados quanto à suavização da componente de tendência observada 9 . Os modelos de Box-Jenkins ajustados a partir de séries diferenciadas são denominados Auto-RegressivosIntegrados-Média-Móveis (ARIMA) com parâmetros "p", “d” e "q", nos quais, "p" e "q" representam o número de observações passadas, e “d”, o número de diferenças realizadas na série.

Neste trabalho, a notação utilizada para representação dos parâmetros do modelo de BoxJenkins foi a seguinte:

- $\mathrm{Z}_{\mathrm{t}}$ : para representar a quantidade de APACOncologia apresentada em um determinado mês " $t$ " observado;

- $\mathrm{Z}_{\mathrm{t}-\mathrm{i}}$ : para representar a quantidade de APACOncologia apresentada em meses anteriores ao mês "t" observado, com i variando de 1 até $n-1$; - $\mathrm{a}_{\mathrm{t}}$ : para representar uma componente aleatória que o conjunto de dados observados não consegue explicar. Essa componente é calculada considerando:

$$
a_{t}=Z_{t}-\hat{Z}_{t}
$$

na qual, $Z_{t}$ representa a quantidade apresentada de APAC-Oncologia em um determinado mês " $t$ ', e $\hat{Z}_{t}$ representa a quantidade esperada de APACOncologia para um determinado mês "t".

O módulo ETS (Estimation Time Series), do pacote estatístico SAS versão 8.2 (SAS Institute, Cary, Estados Unidos), foi utilizado para identificação, ajuste e escolha do modelo de BoxJenkins mais adequado a cada série.

\section{Resultados}

Os dois municípios apresentam-se similares quanto à distribuição percentual de casos novos de câncer e de óbito, para os principais tipos de tumores, e por gênero, apesar de o Rio de Janeiro apresentar população e taxa bruta de incidência de câncer maior do que Belo Horizonte. A produção de quimioterapia e radio- 
terapia dos dois municípios é também similar tanto para a quantidade total de procedimentos quanto para os percentuais por tipo de procedimento, apesar de o Rio Janeiro ter um número maior de unidades prestadoras de serviço, sendo $69 \%$ dessas públicas (Tabela 1 ).

A Figura 1 apresenta as séries históricas de quantidade de APAC-Oncologia de cada município, estratificadas por tipo de tratamento (quimioterapia e radioterapia). Para essas séries, é possível perceber que:

- as séries de quimioterapia de ambos os municípios apresentam tendência de crescimento no período observado. No início da implantação do sistema, o Rio de Janeiro apresentava produção inferior à observada em Belo Horizonte. A diferença observada entre os dois municípios foi sendo reduzida ao longo do período, tendo o Rio de Janeiro superado a produção de Belo Horizonte nos dois últimos anos da série (Figura 1a);

- a série de radioterapia de Belo Horizonte apresenta estabilidade temporal, com uma média 621 APAC-Oncologia de radioterapia por mês. É possível perceber que a série de radioterapia do Rio de Janeiro apresenta maior variabilidade do que a série de Belo Horizonte e, também, uma tendência de crescimento ao longo do período (Figura 1b).

A Figura 2 ilustra as séries padronizadas da produção de APAC-Oncologia estratificadas por tipo de procedimento e prestador de serviço para ambos os municípios, nas quais, observa-se que:

- existe uma tendência maior de crescimento da produção de quimioterapia nos períodos iniciais (novembro de 1998 a maio de 2001) para os prestadores públicos. A partir daí, a tendência de crescimento é semelhante, independente do tipo de prestador analisado (Figura 2a);

- existe uma tendência de crescimento para ambos os tipos de prestadores. A série dos prestadores contratados apresenta tendência de alta até outubro de 2002, retornando, a partir de novembro de 2002, ao mesmo patamar de produção observado em fevereiro de 2002 (Figura 2b); - a série de produção de radioterapia de Belo Horizonte apresenta-se estável, destacando-se o fato de que essa produção, em Belo Horizonte, é realizada apenas pelos prestadores contratados (Figura 2c);

- existe uma tendência de crescimento da produção de APAC-Oncologia para ambos os tipos de prestadores, ocorrendo, contudo, uma maior estabilidade na série de produção do prestador público (Figura 2d).

A análise das funções FAC e FACP para todas as séries (Figura 2) indicou tendência de crescimento em todas as séries de produção. Para retirar ou suavizar essa tendência, optouse por tomar diferenças sucessivas dos valores da própria série. A análise das FAC e FACP das séries diferenciadas indicou que o procedimento foi suficiente para suavizar a componente de tendência observada nas séries originais. O resultado final do processo de ajuste das séries aos modelos de Box-Jenkins encontra-se representado na Tabela 2 , no qual podese destacar que:

- Modelo 1: a série de quimioterapia do Rio de Janeiro ajusta-se a um modelo auto-regressivo com parâmetro 3 - AR(3). Esse modelo indica que, para determinar a tendência da série, são necessários três meses de observação. $\mathrm{O}$ modelo para o prestador público (Modelo 1A) é semelhante ao do município como um todo. No entanto, o prestador contratado apresenta dependência temporal menor, pois se ajusta a um modelo AR(2), como mostra o Modelo 1B;

- Modelo 2: a série de radioterapia do Rio de Janeiro ajusta-se a um modelo ARMA $(3,1)$ - auto-regressiva e média-móvel com parâmetros 3 e 1. O ajuste desse modelo indica que apenas as observações passadas não são suficientes para explicar o atual padrão de tendência da série, pois existem fatores aleatórios que o conjunto de dados considerados não consegue explicar. Observa-se que o padrão geral de formação da série do município é determinado pelo prestador público (Modelo 2A). O prestador contratado (Modelo 2B) apresenta estrutura AR(1), o que denota uma menor dependência temporal entre seus valores;

- Modelo 3: a série de quimioterapia de Belo Horizonte apresenta estrutura de tendência AR(1), o mesmo ocorrendo para ambos os tipos de prestadores de serviço (Modelo 3A e 3B);

- Modelo 4: a série de radioterapia de Belo Horizonte apresenta estrutura de tendência $\mathrm{AR}(2)$, sendo toda a produção oriunda de prestadores contratados.

A partir desses resultados, verifica-se que o padrão de registro da produção de quimioterapia e radioterapia no Município do Rio de Janeiro (Modelo 1 e 2) encontra-se significativamente associado à produção de até três meses passados. Verifica-se também que o padrão de registro dos dados observados no município se caracteriza pela produção das unidades públicas (Modelo 1A e 2A). Em Belo Horizonte, o padrão de registro da produção de quimioterapia (Modelo 3) está associado à produção do mês anterior, enquanto que o padrão de radioterapia (Modelo 4) está associado à produção de dois meses anteriores. 
Tabela 1

Características demográficas, epidemiológicas e de produção dos dois municípios.

\begin{tabular}{|c|c|c|c|c|}
\hline \multirow{2}{*}{ Variáveis } & \multicolumn{2}{|c|}{ Rio de Janeiro } & \multicolumn{2}{|c|}{ Belo Horizonte } \\
\hline & $\mathrm{N}$ & $\%$ & $\mathrm{~N}$ & $\%$ \\
\hline População* & 5.988 .642 & - & 2.294 .226 & - \\
\hline \multicolumn{5}{|c|}{ Taxa bruta de incidência de câncer por gênero* } \\
\hline Geral & 427,92 & - & 315,52 & - \\
\hline Feminina & 440,84 & - & 326,03 & - \\
\hline Masculina & 413,31 & - & 303,78 & - \\
\hline \multicolumn{5}{|c|}{$\begin{array}{l}\text { Estimativa do número de casos novos } \\
\text { para os principais tumores* }\end{array}$} \\
\hline Geral & 25.630 & - & 7.240 & - \\
\hline Pele não melanoma & 3.740 & 15 & 1.300 & 18 \\
\hline Mama & 3.800 & 15 & 860 & 12 \\
\hline Traquéia, brônquios, pulmões & 1.860 & 7 & 380 & 5 \\
\hline Estômago & 1.110 & 4 & 400 & 6 \\
\hline Colo do útero & 970 & 4 & 300 & 4 \\
\hline Próstata & 2.060 & 8 & 600 & 8 \\
\hline Cólon e reto & 1.900 & 7 & 380 & 5 \\
\hline Esôfago & 370 & 1 & 210 & 3 \\
\hline Leucemia & 500 & 2 & 180 & 2 \\
\hline Boca & 950 & 4 & 210 & 3 \\
\hline Pele melanoma & 260 & 1 & 70 & 1 \\
\hline Outras localizações & 8.110 & 32 & 2.350 & 32 \\
\hline \multicolumn{5}{|l|}{$\begin{array}{l}\text { Quantidade de óbitos por câncer } \\
\text { para os principais tumores**}\end{array}$} \\
\hline Geral & 10.213 & - & 3.427 & - \\
\hline Traquéia, brônquios, pulmões & 1.447 & 14 & 315 & 9 \\
\hline Mama & 1.001 & 10 & 261 & 8 \\
\hline Cólon e reto & 912 & 9 & 230 & 7 \\
\hline Estômago & 687 & 7 & 270 & 8 \\
\hline Próstata & 607 & 6 & 197 & 6 \\
\hline Boca & 483 & 5 & 126 & 4 \\
\hline Leucemia & 390 & 4 & 165 & 5 \\
\hline Outras localizações & 4.686 & 46 & 1.863 & 54 \\
\hline \multicolumn{5}{|c|}{ Quantidade e tipo de prestadores ${ }^{\star \star \star}$} \\
\hline Geral & 13 & - & 9 & - \\
\hline Contratado & 4 & 31 & 7 & 78 \\
\hline Público & 9 & 69 & 2 & 22 \\
\hline \multicolumn{5}{|c|}{$\begin{array}{l}\text { Quantidade e tipo de procedimentos } \\
\text { registrados por tipo de unidade } \mathrm{e}^{\star \star \star}\end{array}$} \\
\hline Geral & 265.674 & - & 281.255 & - \\
\hline Quimioterapia geral & 232.183 & 87 & 248.961 & 89 \\
\hline Unidade contratada & 6.034 & 3 & 217.695 & 87 \\
\hline Unidade pública & 226.149 & 97 & 31.266 & 13 \\
\hline Radioterapia geral & 33.491 & 13 & 32.294 & 11 \\
\hline Unidade contratada & 12.464 & 37 & 32.294 & 100 \\
\hline Unidade pública & 21.027 & 63 & 0 & 0 \\
\hline
\end{tabular}

* Estimativas de incidência e mortalidade por câncer no Brasil.

(http://www.inca.gov.br, acessado em 26/Mai/2005).

** Sistema de Informação sobre Mortalidade.

(http://tabnet.datasus.gov.br/cgi/sim/obtmap.htm, acessado em 26/Mai/2005)

*** Sistema de Informação Ambulatorial das Autorizações de Procedimentos de Alta Complexidade.

(http://tabnet.datasus.gov.br/cgi/sia/pamap.htm, acessado em 26/Mai/2005). 
a) Quimioterapia

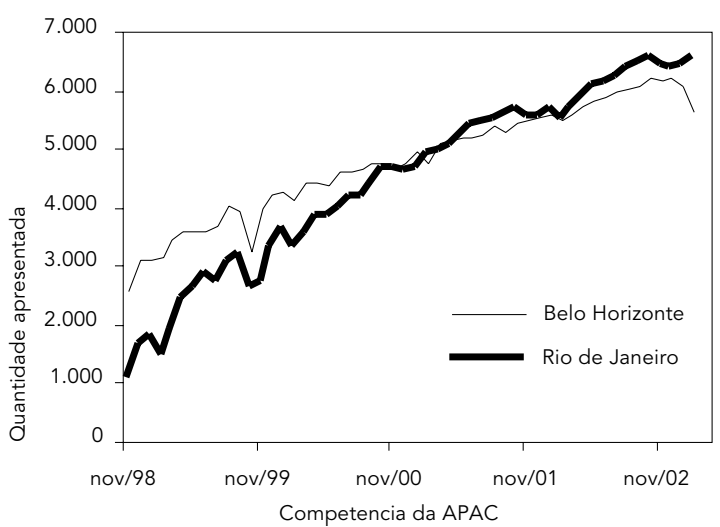

b) Radioterapia

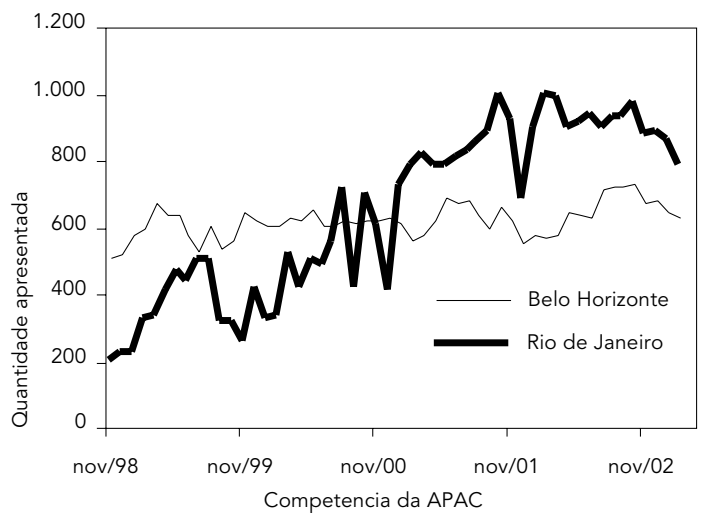

\section{Discussão}

As séries históricas de produção de quimioterapia (Figura 1a) mostraram que ambos os municípios apresentam uma tendência de crescimento da produção. Essa tendência é maior para o Rio de Janeiro, que, apesar de ter iniciado com um menor volume de produção, finalizou o período de observação com valores superiores aos observados para Belo Horizonte. Para a radioterapia (Figura 1b), no entanto, as séries apresentam características bem distintas, sendo a produção de Belo Horizonte relativamente estável e a do Rio de Janeiro mantendo, além de muita irregularidade no padrão, tendência de crescimento. Contudo, no final da série, esse padrão perde força, o que pode ser um reflexo do empenho das unidades em aprimorarem os seus sistemas de faturamento. A tendência de alta nas séries de produção, em parte, pode ser explicada pela necessidade de as unidades se adaptarem ao sistema de faturamento do APACSIA/SUS. As Figuras 2 a e $2 d$ sugerem que está dificuldade foi maior para as unidades públicas do que para as contratadas, contudo, essa diferença não é tão claramente observada na Figura 2b.

Os modelos de Box-Jenkins (Tabela 2) permitiram uma análise mais detalhada do padrão de registro de produção para cada uma das séries descritas acima. Identificou-se uma dependência temporal de três meses, para as séries de quimioterapia e de radioterapia das unidades públicas do Rio de Janeiro; dois meses, para as séries de quimioterapia de unidades contratadas do Rio de Janeiro e de Belo Horizonte; e de um mês, para a série de radioterapia de unidade contratada do Rio do Janeiro e de quimioterapia para as unidades públicas e contratadas de Belo Horizonte (Tabela 2). Dessa forma, os modelos confirmam as hipóteses obtidas na descrição das séries quanto ao maior atraso do registro da produção do Rio de Janeiro, em especial, para as unidades públicas. A dependência máxima obtida nos modelos coincide com o prazo máximo de faturamento das APAC-Oncologia, que é de três meses. Assim, o máximo de tempo que uma unidade pode atrasar é de três meses, o que ocorre para as unidades públicas do Rio de Janeiro. Cabe considerar que as unidades públicas do Rio de Janeiro, credenciadas para prestarem assistência oncológica, são instituições de ensino e pesquisa. $\mathrm{O}$ maior atraso observado por parte dessas unidades é lamentável, pois deveriam ser essas as unidades que melhor respondessem ao sistema, uma vez que os responsáveis pelo serviço são docentes envolvidos em ensino e pesquisa, potencialmente os grandes interessados em dispor de informação consistente para monitorar a assistência prestada e validar suas pesquisas clínicas.

Além do maior atraso, a série de produção de radioterapia das unidades públicas do Rio 
Série histórica padronizada da quantidade de APAC-Oncologia por tipo de tratamento e tipo de unidade prestadora de serviço por município de análise, período de novembro de 1998 a fevereiro de 2003.

a) Quimioterapia, Belo Horizonte

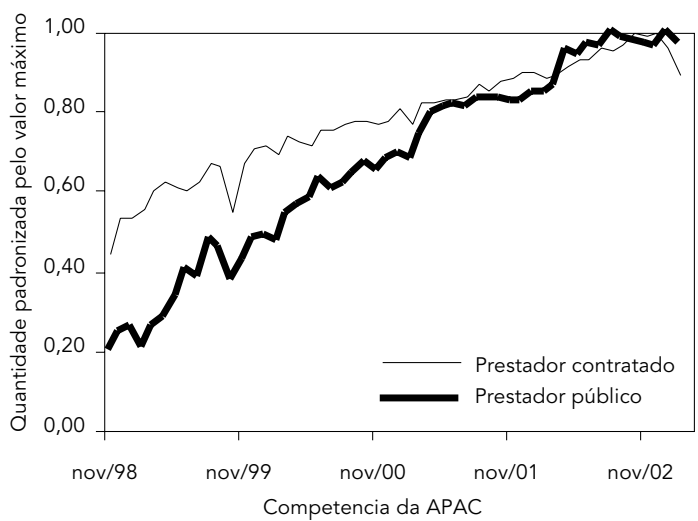

c) Radioterapia, Belo Horizonte

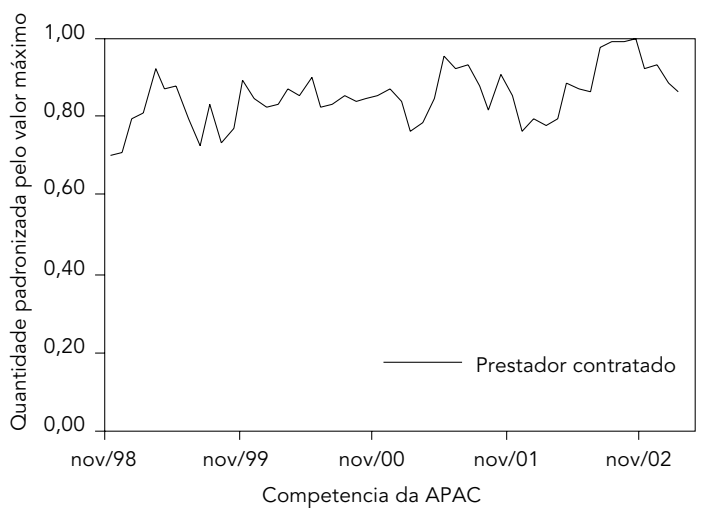

b) Quimioterapia, Rio de Janeiro

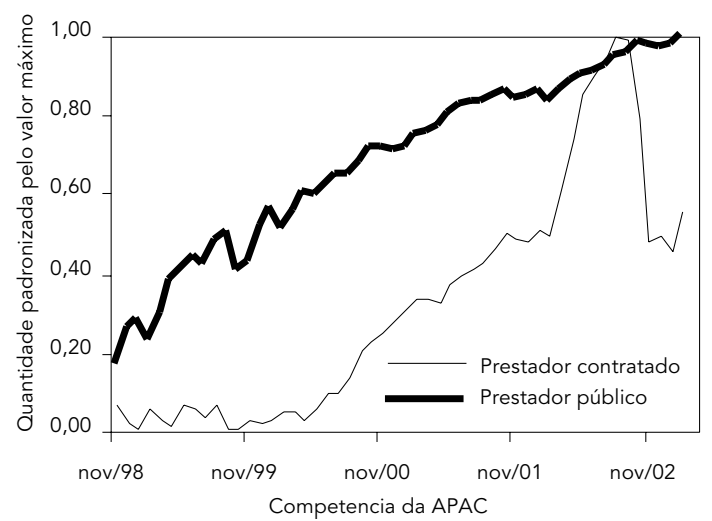

d) Radioterapia, Rio de Janeiro

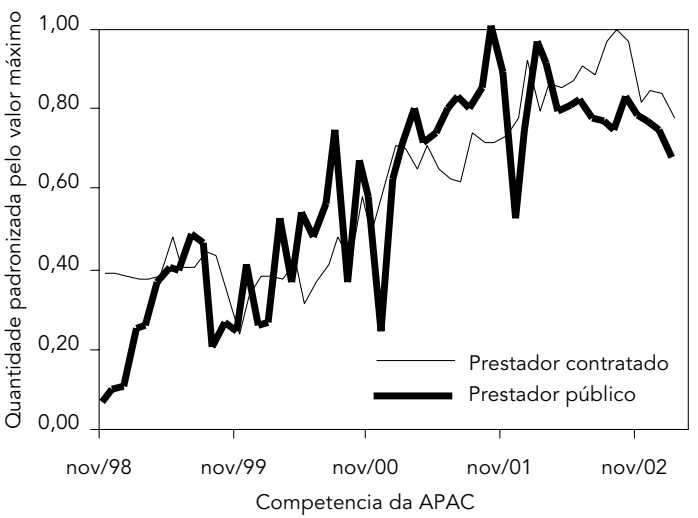

de Janeiro tem um comportamento aleatório predominante, isso pode estar indicando que as unidades prestadoras de serviço ou têm problemas constantes nas condições de assistência (por exemplo: quebra de equipamento, falta de suprimentos ou de recursos humanos) ou no faturamento de sua produção. Nas Figuras $1 b$ e $2 d$, podem ser observadas quedas abruptas no registro da produção para alguns meses, em especial, para o mês de novembro de 2001, quando a queda foi de $50 \%$, tendo a série, a partir daí, desacelerado o padrão de crescimento. Cabe considerar que o padrão geral de registro das unidades públicas do Rio de Janeiro é determinado a partir de um único prestador, que, sozinho, responde por mais de $60 \%$ de toda a produção. Assim, uma interrupção na produção ou no faturamento desse prestador é rapidamente refletida no padrão da série.

Para Belo Horizonte, os modelos não identificaram diferenças significativas no padrão de registro dos dados de produção por parte dos prestadores de serviços. Isso, em parte, deve-se ao fato de a produção estar concentrada nos prestadores contratados, independente do tipo de procedimento. Contudo, o padrão de registro de produção de radioterapia das unidades contratadas em Belo Horizonte apresenta uma 
Tabela 2

Modelos de Box-Jenkins para as séries históricas de produção de quimioterapia e radioterapia para os municípios do Rio de Janeiro e Belo Horizonte, Brasil.

\begin{tabular}{|c|c|c|c|}
\hline Procedimento & Modelo & Rio de Janeiro & Belo Horizonte \\
\hline \multirow[t]{6}{*}{ Quimioterapia } & Todas as unidades & Modelo 1 & Modelo 3 \\
\hline & & $Z_{t}=105,35+Z_{t-1}-0,5 Z_{t-2}+0,5 Z_{t-3}$ & $Z_{t}=60,47+Z_{t-1}$ \\
\hline & Unidade pública & Modelo 1A & Modelo 3A \\
\hline & & $Z_{t}=101,84+Z_{t-1}-0,5 Z_{t-2}+0,5 Z_{t-3}$ & $Z_{t}=13,57+Z_{t-1}$ \\
\hline & Unidade contratada & Modelo 1B & Modelo 3B \\
\hline & & $Z_{t}=3,41+1,41 Z_{t-1}-0,4 Z_{t-2}$ & $Z_{t}=46,90+Z_{t-1}$ \\
\hline \multirow[t]{6}{*}{ Radioterapia } & Todas as unidades & Modelo 2 & Modelo 4 \\
\hline & & $Z_{t}=12,45-0,13 Z_{t-1}-1,68 Z_{t-2}-0,55 Z_{t-3}+a_{t}+0,77 a_{t-1}$ & $Z_{t}=612,09+1,64 Z_{t-1}-1,64 Z_{t-2}$ \\
\hline & Unidade pública & Modelo 2A & Sem produção para este procedimento \\
\hline & & $Z_{t}=9,17-0,82 Z_{t-1}-0,47 Z_{t-2}+0,29 Z_{t-3}+a_{t}-0,37 a_{t-1}$ & \\
\hline & Unidade contratada & Modelo 2B & Igual ao modelo para todas as unidades \\
\hline & & $Z_{t}=3,08+Z_{t-1}$ & \\
\hline
\end{tabular}

dependência temporal maior do que a observada para as unidades contratadas do Rio de Janeiro, só que com uma tendência inversa. Em Belo Horizonte, a produção de radioterapia no mês atual decresce com o aumento da produção nos dois meses anteriores. Isso pode ser conseqüência do fato de os prestadores já terem atingido os seus tetos de faturamento e controlarem o registro segundo esse teto, mostrando, assim, um certo monitoramento da produção ou, pelo menos, do registro dessa, para minimizar a ocorrência de glosa.

As diferenças observadas no padrão de registro dos dados de produção entre os dois municípios podem ter explicação a partir de uma série de fatores, entre os quais, destaca-se a organização local dos serviços de cada município. O "melhor desempenho" observado para Belo Horizonte no registro dos dados de produção pode estar, na realidade, sendo reflexo da participação desse município no Programa de Avaliação e Controle em Oncologia e da existência, desde 1993, de uma política de avaliação e controle da assistência prestada à população. Santos 6 descreve que, dentre os principais efeitos dessa política de controle e avaliação da produção, estariam a melhoria no processo de reembolso da produção e a redução do número de glosas devido a erros de preenchimento dos formulários de controle.

O maior atraso no envio de dados das unidades públicas do Rio de Janeiro pode estar, na realidade, refletindo um outro problema discutido anteriormente por Soares et al. 13, que é a dificuldade que as unidades públicas do Rio de Janeiro apresentam em documentar a produção realizada. Esses autores avaliaram o SIH/SUS (Sistema de Informação Hospitalar do SUS) e identificaram que as unidades públicas enfrentavam problemas na documentação da produção. Entre os motivos apontados pelos autores para essa dificuldade, estava a falta de treinamento do quadro de funcionários dos hospitais para o preenchimento das guias de produção. Como o faturamento de APAC-Oncologia nos hospitais públicos, muito provavelmente, é realizado pelo mesmo grupo de funcionários responsável pelo faturamento do SIH/SUS, podese supor que os problemas detectados anteriormente estejam sendo replicados no faturamento da APAC-Oncologia.

A dificuldade que as unidades públicas do Rio de Janeiro apresentam em registrar a produção realizada também pode estar ajudando a explicar a baixa produção informada por esse município. Os dois municípios apresentam um volume semelhante de produção, o que não se justifica dado que o Município do Rio de Janeiro apresenta maior população e taxa bruta de incidência de câncer do que Belo Horizonte. Assim, para atender a sua demanda local por procedimentos de quimioterapia e radioterapia, deveria ter uma produção significativamente superior, uma vez que tem um perfil epidemiológico semelhante ao de Belo Horizonte (Tabela 1). Esses dados podem levantar as seguintes indagações: “Teria o Município do Rio de Janeiro uma demanda reprimida maior do que a de Belo Horizonte?", “Estariam as estimativas de incidência de câncer superestimadas para o município" ou "Estariam as unidades com um padrão de registro no sistema APAC- 
SIA/SUS muito aquém do que deveriam?". Pode ser que as três situações estejam ocorrendo simultaneamente, contudo, só é possível discutir a última indagação. A tendência de alta, mesmo no final da série de produção do município, indica que o padrão de registro de produção não encontrou estabilidade. Assim, é muito provável que uma parcela dos casos tratados no município não esteja sendo registrada no sistema. Esses achados corroboram com o estudo realizado por Gadelha 14, que mostrou que as unidades que prestam assistência oncológica no Município do Rio de Janeiro apresentam um registro para a produção muito aquém da capacidade instalada.

Finalmente, ressalta-se que a implementação de um sistema de informação não se faz apenas com a aquisição de equipamentos e programas específicos, e sim por um conjunto

\section{Resumo}

A resposta dos serviços à implantação de um sistema de informação depende de uma série de fatores, entre os quais, destaca-se a organização local. Este trabalho comparou o padrão de registro dos dados de produção de quimioterapia e radioterapia dos municípios do Rio de Janeiro e Belo Horizonte, Brasil, no Sistema de Informação Ambulatorial do SUS, no período de novembro de 1998 a fevereiro de 2003. Ambos os municípios mostraram uma tendência de alta na série de produção de quimioterapia, e apenas o Rio de Janeiro, na série de radioterapia. Comparando os modelos de Box-Jenkins de cada série, verificou-se que o Rio de Janeiro apresenta um atraso de três meses no registro de produção, devido às unidades públicas. Em Belo Horizonte, o atraso é menor e não foi observada diferença no registro da produção entre as unidades públicas e contratadas. A diferença observada entre os municípios parece estar associada ao fato de Belo Horizonte dispor de um sistema de controle e avaliação da produção anterior a 1998, ano da implantação do sistema.

Série de Tempo; Sistemas de Informação em Atendimento Ambulatorial; Oncologia de atividades que envolvem desde o treinamento até a adequação do referido sistema ao contexto sócio-regional ao qual este se insere, bem como, organizar o contexto para a implantação de um sistema. Essas atividades fazem parte do que se conceitua como plano estratégico de um sistema de informação 15 , que deve ser permanentemente monitorado para verificar o alcance dos objetivos. Nesse sentido, este estudo contribui para o desenvolvimento de métodos de avaliação do padrão de registro da produção, uma vez que a melhor compreensão desse padrão não só é útil para quem deseja usar esses dados em outras análises, mas, principalmente, para apoiar os gestores municipais e das unidades prestadoras de serviço no acompanhamento do desempenho dessas unidades no registro de sua produção.

\section{Colaboradores}

S. C. S. Gomes Jr. contribuiu na construção e manipulação da base de dados, no desenvolvimento dos modelos teóricos e na análise e discussão dos resultados. R. T. Almeida contribuiu na validação dos modelos teóricos e, também, na análise e discussão dos resultados. Ambos os autores contribuíram na redação final do trabalho.

\section{Agradecimentos}

Trabalho realizado com apoio financeiro recebido do Conselho Nacional de Desenvolvimento Científico e Tecnológico (CNPq), processo 551279/01 e concessão de bolsa ao autor principal pela Coordenação de Aperfeiçoamento de Pessoal de Nível Superior (CAPES) e CNPq. 


\section{Referências}

1. Kligerman J. A assistência oncológica no SUS. Rev Bras Cancerol 1999; 44(4). http://www.inca.gov.br/ $\mathrm{rbc} / \mathrm{n} \_44 / \mathrm{v} 04 /$ editorial.html (acessado em 10/ Nov/2003).

2. Gadelha MIP. Experiências inovadoras em gestão pública federal: como promover e trabalhar em parceria. Rio de Janeiro: Escola Nacional de Administração Pública, Ministério da Administração e Reforma do Estado; 1996.

3. Secretaria de Atenção à Saúde, Ministério da Saúde. Bases técnicas para autorização de procedimentos de alta complexidade APAC-Oncologia. http://dtr2001.saude.gov.br/sas/decas/SUSManual\%209a\%20Edicão\%20-\%20Setembro2003.pdf (acessado em 06/Fev/2004).

4. Kligerman J. Registro hospitalar de câncer no Brasil. Rev Bras Cancerol 2001; 47(4). http://www. inca.gov.br/rbc/n_47/v04/editorial.html (acessado em 27/Mai/2003).

5. Friedman CP, Wyat CJ. Evaluation methods in medical informatics. New York: Springer-Verlag; 2000.

6. Santos FP. O controle e avaliação no nível local: a experiência local de Belo Horizonte. In: Mendes EV, organizador. A organização da saúde no nível local. São Paulo: Editora Hucitec; 1998. p. 197-212.

7. Instituto Nacional de Câncer. Diagnóstico e tratamento. Onde tratar no INCA. http://www.inca. gov.br/conteudo_view.asp?id=1 (acessado em 24/ Out/2005).
8. Gomes Jr. SCS, De Martino R, Almeida RT. Rotinas de integração das tabelas do sistema de autorização de procedimentos de alta complexidade em oncologia do Sistema Único de Saúde. Cad Saúde Colet (Rio J) 2003; 11:231-54.

9. Moretin PA, Toloi CMC. Previsão de séries temporais. São Paulo: Atual Editora; 1985.

10. Helfenstein U. Box-Jenkins modelling in medical research. Stat Methods Med Res 1996; 5:3-22.

11. Costa Neto PLO. Estatística. São Paulo: Edgard Blücher; 1977.

12. Matos OC. Econometria básica: teoria e aplicações. São Paulo: Atlas; 1995.

13. Soares MF, Costa ME, Coeli CM. Avaliação do processo de produção de dados do Sistema de Informações Hospitalares do Sistema Único de Saúde (SIH/SUS) nas unidades públicas de saúde da área de planejamento 2.2. Caderno Politécnico $2000 ; 1: 29-38$.

14. Gadelha MIP. Planejamento da assistência oncológica: um exercício de estimativas. Rev Bras Cancerol 2002; 48:533-453.

15. Winter AF, Ammenwerth E, Bott OJ, Brigl B, Buchauer A, Graber S, et al. Strategic information management plans: the basis for systematic information management in hospitals. Int J Med Inform 2001; 64:99-109.

Recebido em 07/Out/2004

Versão final reapresentada em 07/Jun/2005

Aprovado em 21/Jun/2005 